\title{
Colorantes y pigmentos en las Pesquerías Hispanorromanas
}

\author{
Darío BERNAL-CASASOLA. \\ Universidad de Cádiz. Departamento de Historia, Geografía y Filosofía (dario.bernal@uca.es) \\ Salvador DOMÍNGUEZ-BELLA. \\ Universidad de Cádiz. Departamento de Ciencias de la Tierra (salvador.dominguez@uca.es)
}

A Manuel Bendala, por abrir nuevas sendas en la arqueología
hispanorromana y por impregnarnos
durante años con su magisterio

\begin{abstract}
Resumen
Se presenta un aspecto poco tratado por la investigación, cual es la presencia, uso y fabricación de colorantes minerales -y posiblemente de otra naturaleza- en las fábricas de salazón hispanorromanas. A raíz de la detección de cinabrio machacado en molinos manuales en las cetariae de la antigua lulia Traducta (actividad arqueológica preventiva en la c/ San Nicolás de Algeciras, Cádiz) se plantean los posibles usos de estos pigmentos en la industria pesquero-conservera, conjuntamente con otras evidencias arqueológicas, etnográficas y literarias. Básicamente su empleo para tintar las conservas de pescado y hacerlas más atractivas para los clientes, para endurecer las redes y garantizar su perdurabilidad, para realizar los tituli picti de las ánforas (rubrum) o para la pesca con venenos, bien atestiguada en las fuentes clásicas. Todo ello constituye una novedosa línea de investigación a desarrollar en los próximos años, tratando con estas páginas de llamar la atención sobre la necesidad de valorar la complejidad de estos procesos artesanales en las industrias haliéuticas de la Hispania romana.
\end{abstract}

Palabras clave: Pesca, Industria conservera, Colorantes, Pigmentos, Cinabrio, Época Romana, Baetica.

\section{Summary}

This paper deals with an aspect no well treated by research, which is the presence, use and manufacture of mineral colourings - and possibly vegetal substances - in the fish processing plants in ancient Hispania. The detection of cinnabar grains in hand-mills found at the Iulia Traducta cetariae (rescue dig at Saint Nicholas Street, Algeciras, Cádiz) has led us to think about the potential uses of these pigments in the fishing-salting industry, analyzing together with other archaeological, ethno-graphical and literary evidences their different uses in Antiquity. Mainly their employment to dye fish-preserves to make them more attractive for the clients; to harden the networks and other fishing gear and to guarantee their preservation; to draw the commercial tituli picti of the amphorae (rubrum); or maybe also used for fishing with poisons, well testified practice in the classical sources. All this constitutes a new line of research to be developed in the next years, trying with these pages to call the attention on the need to value the complexity of the handcrafted processes carried out in the halieutic sites of Roman Spain.

Keywords: Fishing, Fish Processing, Dyes, Pigments, Cinnabar, Roman Times, Baetica. 


\section{INTRODUCCIÓN ${ }^{1}$}

El ciclo de la pesca y la industria conservera hispanorromana está bien definido desde los pioneros estudios de M. Ponsich y M. Tarradell, investigadores que fijaron sabiamente tanto las diferentes etapas en los procesos artesanales de elaboración de conservas piscícolas -de las capturas a la elaboración de salsamenta-, a los escasos indicadores arqueológicos que de dichos procesos nos han quedado en el registro - saladeros comúnmente llamados "fábricas de salazón", alfarerías para las ánforas salsero-salazoneras y salinae-, guiados de la mano de un acercamiento interdisciplinar (Ponsich, 1988, 27-65). Desde los años sesenta -fecha de edición de la monografía que permitió la definición de esta nueva parcela de la Arqueología de la Producción hispanorromana, titulada Garum et industries antiques de salaison dans la Méditerránee Occidentale-, el conocimiento de nuestras cetariae ha ido in crescendo, como se advierte en las principales obras de síntesis, como en la clásica compilada por F. Mayet (Étienne y Mayet, 2002). En este último trabajo, que recoge la tradición y el savoir faire que hoy llamaríamos "post-ponsichniano" queda patente cómo el acercamiento a los recursos bióticosabióticos -sal, pescado, tipos de pesca...- es totalmente teórico por falta de información arqueológica, limitándose los estudios a las ánforas y a su amplio aparato epigráfico -lo que permite hablar de la riqueza y variedad de salsas a los mercatores hispánicos- y a las fábricas de salazón, la aproximación a las cuales es eminentemente topográfico-arquitectónica, con sus correspondientes variables cronológica y volumétrica -cálculos de capacidad-.

Esta tendencia se ha mantenido hasta prácticamente nuestros días, centrándose los estudios en la interpretación de las chancas de manera epidérmica, pues prácticamente todas ellas adolecen, como sucedía en época de los eminentes hispanistas citados, de estudios analíticos y de detalle. Desgraciadamente, y muy a nuestro pesar, son mínimas las cetariae hispanas que disponen de una monografía editada en la cual se presenten, de manera analítica e inductiva, y simbióticamente entrelazados, los resultados procedentes de caracterizaciones arqueozoológicas, estudios arqueobotánicos, o estudios integrales del utillaje aparecido en estos centros de producción conservera, como sucede con el instrumental pesquero. Una dinámica que no es, ni mucho menos, atribuible a la investigación hispanorromana, sino a la generalidad de las aproximaciones histórico-arqueológicas a esta parcela, como se constata en otros lugares del Mundo Antiguo, como por ejemplo en el Mar Negro (Bekker-Nielsen, 2003) o en el área suditálica y siciliana (Botte, 2009), que son como es bien sabido, las zonas pesqueras más importantes de nuestra cultura grecorromana, junto al Fretum Gaditanum.

Esta tendencia, justificada en su momento por una Arqueología Clásica entendida por entonces como no necesitada de la recurrencia al dato arqueométrico preciso y a la insustituible información del registro mueble, sigue presente en buena parte de los yacimientos litorales de la Hispania romana, cuyo conocimiento aún es epidérmico y puntual. Desde hace años la aproximación arqueológica a estos ambientes industriales ha cambiado, y de manera lenta pero sin pausa las fábricas hispanorromanas están siendo estudiadas en detalle y desde una óptica interdisciplinar, como sucede, por ejemplo, en Baelo Claudia (Arévalo y Bernal, 2007 eds.) o en complejos haliéuticos como el de Nabeul en el Africa Proconsularis (Slim et alii, 2007), o en general en trabajos de corte temático (García Vargas, 2006). Además del estudio sistemático de las chancas antiguas en los últimos años se han iniciado otras líneas de investigación complementarias, como es el caso del análisis del instrumental pesquero (Bekker-Nielsen y Bernal, 2010 y www.sagena.es), la acuicultura (Bernal, Alonso y Gracia, 2011) y un sinfín de actividades desarrolladas en los centros de procesado piscícola del Estrecho de Gibraltar, desde la producción de púrpura a la elaboración de harina de pescado (Bernal, 2007 y 2009 ed.). La contribución que

Consejería de Innovación, Ciencia y Empresa de la Junta de

Andalucía (HUM-03015, www.sagena.es).
1 Este trabajo se inserta en el marco de desarrollo del proyecto PURPURARIA (HAR2010-15733) del Plan Nacional I+D+i, y del Proyecto de Excelencia SAGENA, de la 
presentamos en estas páginas constituye un resultado de la aplicación metodológica de este tipo de estrategias analíticas y arqueométricas en nuestros yacimientos haliéuticos.

\section{LA DETECCIÍN DE CINABRIO EN LAS CETARIAE DE IULIA TRADUCTA}

Las excavaciones arqueológicas preventivas desarrolladas entre el año 1998 y 2006 en la Villa Vieja de Algeciras han permitido la exhumación de numerosas construcciones interpretadas como pertenecientes al barrio pesquero-conservero de la antigua ciudad augustea de Iulia Traducta: algunas calles que delimitan entre sí insulae destinadas a la elaboración de salsamenta y salsas saladas de pescado (problemática general en Jiménez-Camino y Bernal, 2007). Se han identificado y excavado hasta la fecha un conjunto de tres cetariae en la c/ San Nicolás 1 (Conjuntos A, $\mathrm{B}$ y C/D) y dos en los $n^{\circ} 3-5$ de dicha arteria viaria (Conjuntos I y II), de las cuales se han dado algunos avances (Bernal et alii, 2003), y cuyo estudio monográfico está ultimado, y en imprenta en las fechas de redacción de estas páginas (Bernal, 2012, ed.)

Una de las singularidades de la excavación de estas factorías de salazón, construidas en época augustea pero a pleno rendimiento hasta inicios del s. VI d.C. fue la documentación de una decena de molinos de mano, de tracción rotatoria y de reducidas dimensiones (unos 20-30 cms. de diámetro), aparecidos tanto in situ en algunas de las áreas de trabajo, con la meta encastrada en el pavimento de signinum para facilitar la molturación (figura 1); como en depósitos secundarios, desechados tras su abandono, en los niveles depo-

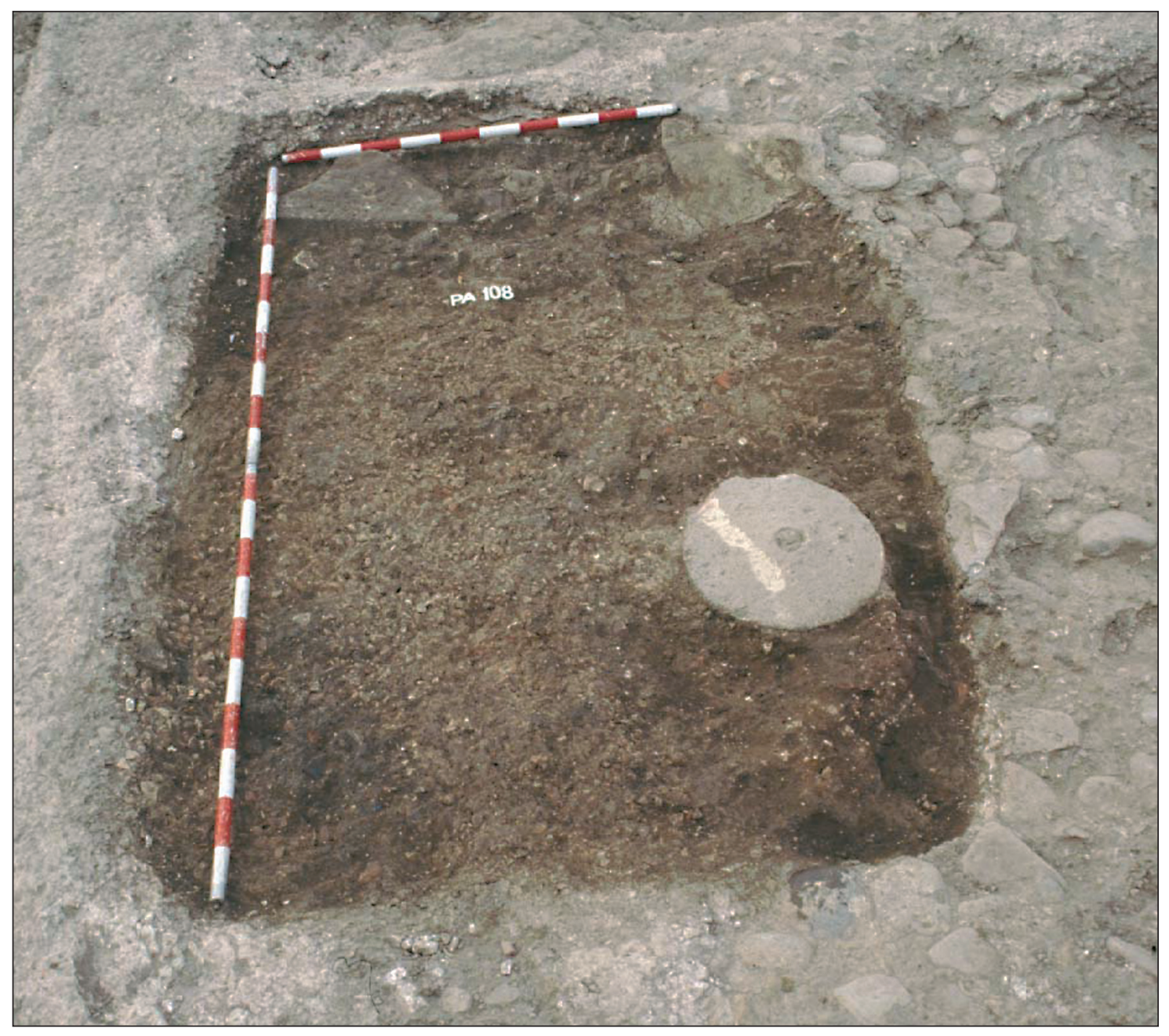

Figura 1.- Parte fija o meta de un molino rotatorio encastrada en el pavimento de opus signinum (PA-108) construido sobre la pileta P-26 del Conjunto Industrial I de la c/ San Nicolás 3-5, cuando dicho ambiente se reconvirtió en área de molturación. 
sicionales de relleno de piletas La hipótesis del empleo de los mismos para el machacado de los huesos de peces para elaborar harina y aceites de pescado (Bernal, 2007) ha podido ser confirmada por analíticas arqueométricas, las cuales han permitido la detección de restos ictiológicos machacados adheridos a los poros de las partes activas de estas herramientas de molturación (Domínguez-Bella y Bernal-Casasola, 2011, 455456). El estudio de los fragmentos seleccionados o de los granos de sedimento procedentes de las partes activas de los molinos, tras su conveniente preparación (tratamiento por ultrasonido, filtrado, triado manual) fue acometido mediante un microscopio estereoscópico y microscopía electrónica de barrido ambiental (ESEM), además de proceder a su caracterización analítica (por EDX y XRD). Los principales resultados, además de determinar la procedencia local/regional de la roca biocalcarenítica utilizada para la elaboración de los molinos -muy similar o idéntica a la llamada localmente "ostionera"- (Gutiérrez Mas et alii, 1991), fueron la identificación de restos microscópicos de escamas y vértebras de ictiofauna, además de espinas de erizos marinos, junto a pequeños micro-granos minerales de color rojo bermellón (Domínguez-Bella, 2012). Además de verificar por ello el machacado de restos ictiológicos y malacológicos para elaborar subproductos piscícolas, el estudio por microscopía y el análisis químico de dichos granos minerales (figura 2), de entre 50 y 70 micras de diámetro, permitió identificar que en las fábricas conserveras algecireñas estaban machacando asimismo cinabrio - $\mathrm{HgS}-$. La identificación de este producto fue de gran interés, al tratarse de un mineral alóctono, quizás procedente del área de Almadén en Ciudad Real, zona geográfica en la cual se conoce la explotación del cinabrio desde la Antigüedad y de forma sistemática en época romana según Plinio (problemática general e identificación en DomínguezBella y Bernal-Casasola, 2011, 456-457).

Conscientes del habitual empleo de este pigmento para la elaboración de pintura mural (Domínguez-Bella, 2004), también en la zona gaditana, ésta era una de las primeras posibilidades interpretativas, si bien la misma no tenía mucho sentido en un contex to haliéutico como el

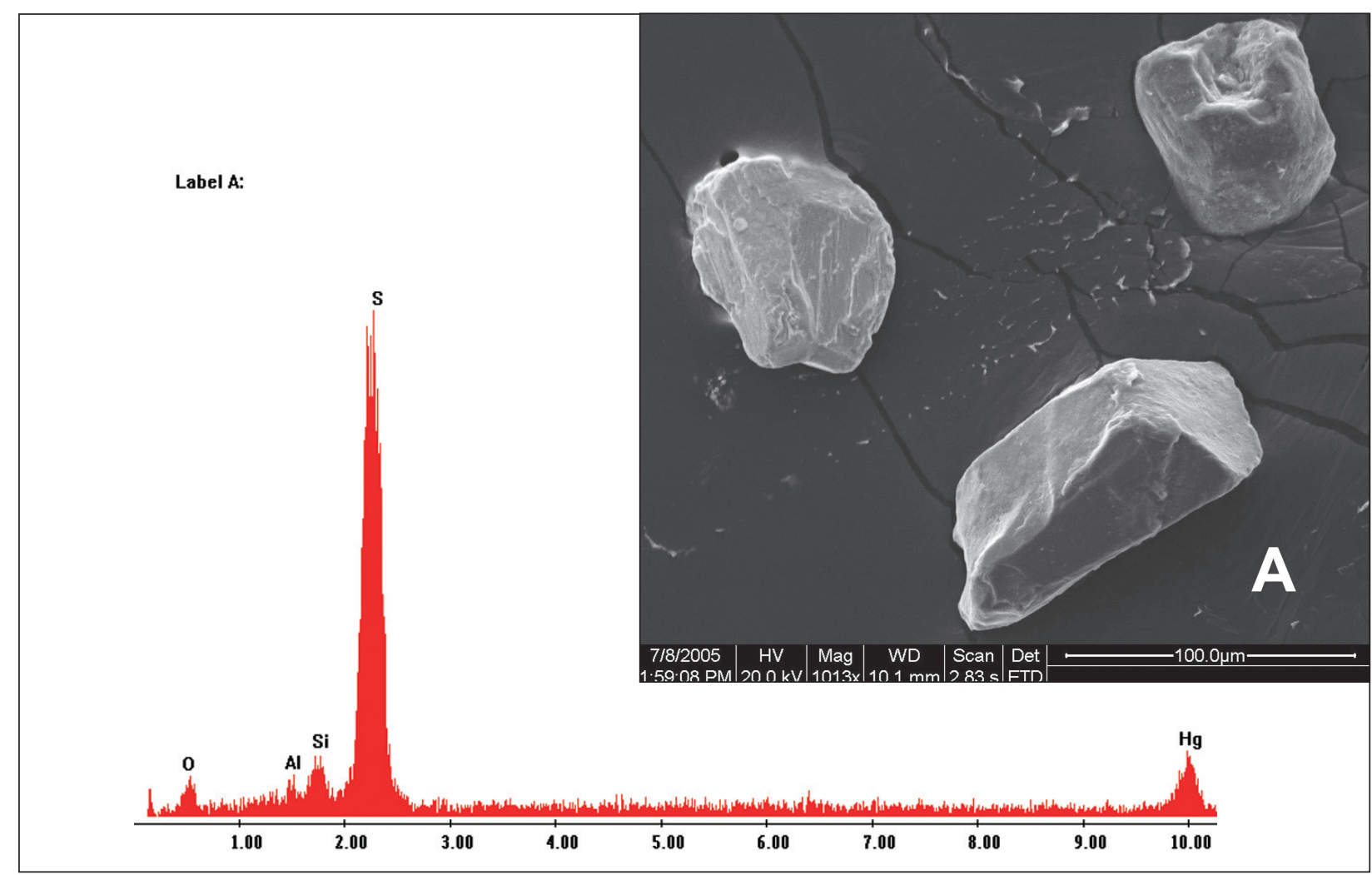

Figura 2.- Vista mediante microscopía electrónica de barrido ambiental (ESEM) de los granos de cinabrio (SHg), recuperados en los molinos (A), y resultados del análisis químico mediante EDS de los mismos con la típica composición del cinabrio (presencia de picos del azufre y del mercurio). 
de su hallazgo: en dos molinos procedentes de una fábrica salazonera, abandonados a finales del s. V o inicios del s. VI d.C. De ahí que también se apuntase en su momento su potencial relación con procesos de conservación y/o elaboración de conservas piscícolas, a pesar del problema de los compuestos venenosos del mercurio que, a priori, complicaban esta línea interpretativa (Domínguez-Bella y Bernal-Casasola, 2011, 457).

Este trabajo constituye el desarrollo del anterior, en el cual se discuten algunos de los potenciales usos de sustancias colorantes en la Antigüedad Clásica, no únicamente del cinabrio, sino del empleo de cualquier sustancia de origen mineral o vegetal como colorante en las actividades de la cadena del ciclo pesquero-conservero. A pesar de que el ínfimo carácter del hallazgo no permite decantarse con claridad sobre su uso en el caso de las cetariae traductenses, la constatación de cinabrio en directa asociación con las fábricas conserveras abre una interesante línea de investigación para el futuro, que estas páginas tratan de estimular.

\section{Colorantes Para las CONSERVAS PISCí- COLAS. ¿UNA PRÁCTICA PARA COMPETIR CON EL HAIMATION?}

Sobre las diversas modalidades de las salsas y derivados de la pesca conocemos mucha aunque fragmentaria y anacrónica literatura, que de manera combinada con la epigrafía pintada en las ánforas para su comercialización ha permitido sintetizar que en las fábricas conserveras se realizaban salsamenta, garum, muria, liquamen, hallec, limphatum o laccatum como productos estrella, cuyo proceso de fabricación está bien identificado y contrastado (una síntesis de todo ello en Étienne y Mayet, 2002, 43-53). Pero la realidad cotidiana debió ser mucho más compleja.

Recientemente se ha propuesto que la clásica interpretación de las inscripciones pintadas COD TI VE o COD LIX VE desarrolladas como Cordula Tingitana/Lixitana Vetus, asociados tradicionalmente a los "atunetes" envejecidos procedentes de Tánger o Lixus, quizás esconda tras de sí la realidad de una salsa piscícola tintada intencionalmente. En Colonia, el hallazgo de un ánfo- ra con la inscripción COD SARD LIX ha sido desarrollada como Cod(ae) Sard(inae) Lix, es decir, una salsa líquida realizada con colas de clupeidos -sardinas- (Ehmig, 2007 a, 272, Cat. N ${ }^{\circ}$ 97; 2007 b, 230). Según esta propuesta, la tradicional interpretación alusiva a la origo mauretana de las salsas podría desarrollarse de otra manera, según la cual TINC/G escondería tras de sí TINCTVS/A en vez de TINGITANA (Ehmig, 2007 a, 230, Cat. $\mathrm{N}^{\mathrm{o}}$ 106). Se ha planteado recientemente una interpretación similar para una Beltrán II A recuperada del depósito portuario durante las actividades arqueológicas de excavación del pecio flavio-adrianeo de Arles-Rhône 3 (Quillon, $2011,108)$. Es decir se trataría de CODAE TINCTAE VETERES, una salsa realizada con colas de peces tintadas y envejecidas. Un producto similar, salvando las distancias, a nuestras sardinas enlatadas en escabeche, aderezadas con pimentón y picantes, es decir tinctae et argutae.

Esta nueva interpretación requiere un sosegado estudio en el futuro, ya que los ríos de tinta vertidos en torno a la importancia de las salsas saladas de pescado de la Tingitana dejarían de existir como tales. Desde hace años se valora el carácter forzado de la transcripción de COD como $\mathrm{CO}(\mathrm{R}) \mathrm{DULA}$, con la necesaria inclusión de una $\mathrm{R}$ intercalada: de las 17 ocasiones en las cuales se registra la citada expresión asociada a los potenciales epítetos mauritanos, en once casos se presenta como COD y solamente en tres de ellos como CORD (Cerri, 2006, 2177-2180, $\mathrm{n}^{\circ} 10,16$ y 23), amén de otras posibilidades (CO o CONDT). $\mathrm{Y}$ en muchas ocasiones es frecuente la mención COD LIX (como por ejemplo en CEIPAC, $\mathrm{n}^{\circ}$ $12020,19749)$, restituible como una salsa confeccionada a base de extremidades caudales de pequeños peces.

Pero no se trata, ni mucho menos, del único caso disponible sobre salsas tintadas. En el volumen de las inscripciones pompeyanas y herculanenses del CIL se propuso el desarrollo de algunas de ellas como Laccat(um) et tinc(tum) vet(us) (CIL, IV, 5642), y en un ejemplar en un muy buen estado de conservación de Pompeya el primer registro del titulus permitía la lectura Laccatum Tinctum (CIL IV, 5641; y similar en IV 9373). Independientemente de la compleja interpretación de la naturaleza en sí misma del laccatum -salsa 
piscícola, bebida a base de leche, un vino o como proponía Dressel vino con la adición de una planta medicinal llamada lacca (problemática general en Curtis, 1991, 8-9, nota 12; Étienne y Mayet, 2002, 52-53)- parece evidente que el producto había sido coloreado con pigmentos vegetales/minerales o "empapado" en algún líquido, si atendemos a la definición etimológica de tinctum. Actualmente algunos autores consideran a este producto como un tipo de salsa piscícola (Ehmig, 2003, 65).

Es difícil desentrañar el verdadero significado del "tintado" en asociación a estas salsas, si bien disponemos de algunos testimonios etnográficos que permiten avanzar al respecto. En las costumbres culinarias de la Antigüedad la importancia de la apariencia y de la forma de presentación de la comida fue notable (Curtis, 2001). Disponemos de un magnífico ejemplo etnográfico procedente de Grecia, que ilustra a la perfección la recurrencia al color rojo como potenciador de la apetencia para el pescado, recientemente recordado por Mylona $(2008,115)$. A inicios del siglo XX en la ciudad de Volos (Tesalia) se empleaba colorante en el proceso de salado de las sardinas, concretamente tierra rica en minerales férricos, que se traía ex profeso en barco desde las Islas Espóradas -sobre todo desde Skopelos- y que se mezclaba con la sal durante el proceso de elaboración de las conservas (Athanassopoulos, 1926). Dicha práctica producía sardinas saladas en un jugo de color rojo intenso, frente a la tradicional carne de color rosa pálido procedente del salado sin colorante, y estos productos eran comercializados sobre todo a Oriente Medio, donde eran muy apreciados. Los análisis oficiales realizados en su momento para determinar las propiedades dietéticas y de sabor organolépticas diríamos hoy- resultado de la adición de tierra roja, demostraron que aparte del cambio del color del preparado, no se producía ninguna otra alteración química perceptible en el alimento. A pesar de ello, los productores conserveros se jactaban de que esta práctica otorgaba a las sardinas en salmuera no únicamente un mejor sabor, sino asimismo un aroma mucho más apetecible (Mylona, 2008, 114).

Es muy probable que un estudio monográfico al efecto depare prácticas similares en otras áreas del Mediterráneo Occidental. Otro tema diferente es el uso de ingredientes que "enrojecen" el preparado piscícola durante su maceración, previamente a su ingestión o tras el salado y su presentación a la mesa, aunque su adición tiene como finalidad aportar un determinado "bouquet" al plato. Así sucede con el empleo de pimentón para el consumo de la mojama o "tonyina de sorra" en el Levante español, o el espolvoreo de pimienta roja molida durante el proceso de salazón de la melva o caballa, como en Denia (Gallart, Escriche y Fito, 2004, 115 y 138).

Quizás una explicación posible al empleo de colorantes rojos para tintar las salsas piscícolas no sea otra que tratar de reproducir el famoso garum haimation o "garo de sangre", realizado con las branquias, el suero y todos los jugos hipogástricos, además de la sangre, que como sabemos por las fuentes era el de mejor calidad (como transmiten, entre otros, los Geoponica en época altomedieval; XX, 46). Esta salsa ha sido identificada arqueológicamente hace unos años en un contexto altoimperial de Jordania, durante su fase de fabricación (Van Neer y Parker, 2008).

\section{ENTINTANDO LAS REDES Y OTROS APAREJOS}

Las redes han sido habitualmente teñidas para evitar su deterioro. A finales del s. XVIII, Sáñez Reguart resume concisamente cómo las "mallas" sufren tras su confección y su primer uso, pues "padece sus alteraciones apenas una red se ha metido en el agua, y mucho más si se tiñe, ya sea con corteza de encina o de otros árboles, o bien si se alquitrana..."; adicionalmente nos recuerda este autor cómo las redes pierden tamaño al tintarse: "después de teñida ya no tiene aquel mismo cuadrado, el cual disminuye una parte de su ámbito con el tinte en caliente, que al secarse hace con precisión encoger el hilo por una demostrable causa física..."; y, por último, nos indica cómo "las redes que se han de alquitranar no se estiran, á efecto de que sus nudos no se aprieten y pueda introducirse con más facilidad el betún por entre sus junturas, con lo que la malla queda, desde luego, minorada. Las redes que se tiñen con cocimiento de corteza, se estiran bien después que se han bañado del tinte, y se observa que los ámbitos o cuadrados no disminuyen tanto..." (Sáñez Reguart, 1793, IV, 95, 96 y 97 respectivamente). 
Todo ello presenta la problemática de que las luces de mallas aparentemente disminuían con el entintado, aunque no fuese así de facto, lo que era aprovechado por pescadores para justificar unos enmalles de mayor tamaño durante su fabricación, en continuo conflicto con las autoridades reguladoras de las pesquerías.

La "entintadura" es el vocablo tradicional usado por pescadores referido al teñido de artes y aparejos para la pesca marítima, e incluso a veces para la fluvial, proceso que ha perdurado hasta bien entrado el s. XX (Rodríguez Santamaría, 1923, 432-433), previamente a la imposición de las fibras sintéticas en el mercado. El objetivo del proceso es variado, y normalmente se las tiñe de color castaño oscuro, para conseguir una mayor durabilidad mediante la reducción de la elasticidad y su desengrase, ya que los fluidos de los peces terminarían por pudrir los aparejos si no se realizase este proceso cíclicamente -varias veces al mes-. En el s. XX sabemos que el proceso, con ciertas variaciones regionales, se hacía cociendo agua dulce en una caldera depositada en estructuras de mampostería, sobre trípodes o incluso encima de piedras. Los productos utilizados varían, desde los polvos de corteza de pino molida o sin moler, corteza de sauce, preparados importados al efecto o incluso alquitrán dulce o vegetal, que se reducían térmicamente en el agua hasta que el producto adquiría la textura adecuada, procediéndose a introducir el aparejo en el envase o verter el contenido en un receptáculo en el cual se colocaba el arte, restando en el líquido varias horas (Rodríguez Santamaría, 1923, 433).

La siguiente pregunta es si este tipo de prácticas también acontecían en la Antigüedad. La información disponible sobre redes en el Mundo antiguo es tremendamente limitada, y aunque etnográficamente conocemos el habitual tintado de las mismas -con paralelos en Ibiza- no está documentada aún esta práctica en los restos orgánicos de redes aparecidos en el Mundo Antiguo (Alfaro, 2010, 81), al menos hasta donde sabemos. Posiblemente se deba, únicamente a la escasez de fibras conservadas de las mismas y a la ausencia de análisis químicos para tratar de aislar colorantes, lo que constituye una interesante línea de investigación para el futuro.
"No podemos decir lo mismo de otros aparejos, como las fibras usadas para la pesca con anzuelo, claramente teñidas en época romana, como nos recuerda Eliano en la Historia de los Animales al indicar: "...si las crines son teñidas, los pescadores eligen las que están teñidas en gris azulado y en púrpura marina....utilizan asimismo los pescadores lana carmesí y lana teñida de púrpura..." (XII, 43).

Un paralelo de gran interés es el constatado en el área de Mazarrón, ámbito geográfico en el cual tradicionalmente se han tintado las redes con polvo de almagra (Martínez e Iniesta, 2007, 69). El hallazgo de una bolsada de almagra en la excavación arqueológica del Callejón de la Sal del Puerto de Mazarrón ha sido interpretado como una evidencia clarividente de cómo en época romana también se tintaban las redes con el mismo producto (Martínez e Iniesta, 2007, 69). En esta actividad arqueológica, de la cual se ha publicado únicamente un avance, se documentaron en el ángulo suroeste del solar intervenido tres piletas y parte de una habitación, de cronología tardía y vinculadas por los excavadores con la industria de salazones de pescado y posiblemente la producción de sal, habiendo sufrido dos momentos de utilización con un abandono intermedio y con su parcial destrucción (Agüera e Iniesta, 1998, 46). No tenemos constancia de que se hayan realizado análisis u otros estudios complementarios al efecto, pero la vinculación de la almagra con las actividades haliéuticas en estos contextos de Mazarrón parece evidente. Otra cuestión es la verificación de su empleo para el entintado de las redes/aparejos o bien como hemos indicado en el apartado precedente para colorear las salsas piscícolas y hacerlas más atractivas para su venta. En cualquier caso, constituye un ejemplo clarividente del empleo de materias colorantes en las fábricas conserveras hispanorromanas, demostrando que el caso de Traducta no es ni único ni aislado, compartiendo adicionalmente con el mismo la cronología tardorromana.

El cinabrio cuenta entre sus propiedades con un notable carácter fungicida, al igual que la almagra (Mahdihassan, 1984; Martín Gil et alii, 1995). De ahí que el empleo de ambas sustancias para evitar la corrosión de las fibras utilizadas para la elaboración de las redes es una hipótesis a tener muy en cuenta. 
Por último, indicar que el sistema tradicional de preparado del colorante para el entintado de las redes ha sido, asimismo, por molturación. Un buen ejemplo lo tenemos en la preparación del colorante de coníferas de color castaño oscuro a negro, hasta las primeras décadas del s. XX: "en el sur de España y en Levante hay puertos de mar que tienen molinos expresamente para la corteza de pino, sin sangrar, que reducen a polvo, exportándolo luego al resto de los puertos del sur de España y de las costas de Levante, que lo reciben en sacos con los nombres de polvos, cáscara y entintadura" (Rodríguez Santamaría, 1923, 433). El sistema de fabricación es similar, por molturación, y quizás constituya éste un reflejo indirecto de las prácticas realizadas en las fábricas salazoneras romanas de Algeciras.

\section{ATRAMENTUm/Rubrum, Pigmentos NECE- SARIOS PARA lOS TITULI PICTI}

Tras el envasado en las ánforas de los tacos salados de carne-salsamenta- o de las salsas piscícolas y su conveniente hermetización con los tapones u opercula, la siguiente operación era el etiquetado de los envases. Un procedimiento comercial tremendamente detallado y muy bien conocido en la Antigüedad para el caso de las ánforas olearias del Valle del Guadalquivir (Dr. 20) gracias, especialmente, al Monte Testaccio, cuya estandarización permite una identificación milimétrica de cada registro en la actualidad (Aguilera, 2004). Desgraciadamente para las ánforas salazoneras béticas el formulario es mucho más parco, a pesar de que el mismo está muy estandarizado y es bien conocido, dividido entre tituli primarios y secundarios (propuesta en Martín Kilcher, 2002; análisis de casos en Lagóstena, 2004). Parece consensuado y aceptado por la comunidad científica que las inscripciones "primarias" (relativas al tipo de producto, a su calidad, cantidad, nombre del negotiator y cognomen bajo el asa) se realizan en los mismos lugares de producción de la salazón, en los cuales se rellenan las ánforas y se procede a la venta al por mayor a los negotiantes salsarii. Es decir, cerca o en las propias cetariae procedería el negotiator directamente o a través de sus representantes a la compra con los productores, probando la mercancía -como en las conocidas degustationes del vino- y dejando verificación epigráfica -cognomi- na bajo el asa- de dicho proceso. Otras inscripciones secundarias, realizadas con posterioridad -por la diversa grafía y por la estratigrafía, al cubrir en algunos casos a las anteriores- son relativas al tránsito comercial y a otras etapas en la vida de la venta de las mercancías (Martín Kilcher, 2002, 345).

Aunque en este proceso está sometido a cierta controversia, pues no siempre es posible interpretar con claridad todas las evidencias epigráficas con cargo al modelo conscientemente simplificado en el párrafo anterior (una buen ejercicio de reflexión, por ejemplo en Chic, 2002), lo que sí parece evidente es que el proceso de verificación de la calidad de las salas, el envasado de las mismas y el precintado de las ánforas se hacía en el ámbito de las chancas litorales. Y en ese momento se pintaban las inscripciones fundamentales o "primarias" de los tituli picti, que son las que nos encontramos habitualmente en los pecios y yacimientos húmedos del ámbito atlántico-mediterráneo, pues las demás son abreviadas y de compleja hermenéutica.

Para la ejecución de dichas inscripciones era necesario disponer de los pigmentos necesarios para su elaboración in situ. Normalmente se utilizaban dos tipos de tintas, una negra, bien conocida como atramentum, y otra de color rojo que habitualmente denominamos rubrum. No tenemos constancia de estudios cuantitativos que reflejen las proporciones del empleo de un tipo $\mathrm{u}$ otro de tinta, si bien la consuetudo induce a pensar que predominan las inscripciones en atramentum sobre las ánforas béticas. No obstante, tenemos constancia del empleo de inscripciones en rojo en prácticamente todos los tipos salsarios béticos, desde las Mañá C2B/Ramon T-7433 (CIL XV, 4730), Dr. 7 (CIL XV, 4713, 4737), Dr. 8 (CIL XV, 4718), Dr. 9 (CIL XV, 4758, 4782), Dr. 10 y 11 (CIL XV, 4726), y numerosos ejemplos en Pompei VII, Beltrán IIA y B (citas precisas en Lagóstena, 2004, 204-216). Es decir, la conclusión no es otra que la necesidad en las cetariae baeticae de disponer del instrumental y los pigmentos necesarios para elaborar este tipo de dipinti. Una práctica que discurre en paralelo al desarrollo de la industria salazonera, desde al menos el s. I a.C. y a lo largo de la totalidad del Alto Imperio hasta al menos finales del s. II d.C., 
como demuestran los tituli en rubrum en los tipos anteriormente citados. Desgraciadamente la escasez de inscripciones en ánforas hispanas tardoantiguas no permite confirmar empíricamente la continuidad de estas prácticas, pues en uno de los escasos ejemplos conocidos no se especifica el color del dipintus (Almagro $51 \mathrm{c}$ de Tossalet en Fernández Izquierdo, 1984, 54, n ${ }^{\circ}$ 153). Continuidad, que a pesar de la ausencia de evidencias, parece la propuesta más lógica y coherente.

Se han realizado varios estudios analíticos sobre el atramentum vinculado a la elaboración de inscripciones sobre ánforas romanas, habiendo podido confirmar que en tal caso los pigmentos eran de origen orgánico, no siendo posible precisar con claridad la identidad de las sustancias, quizás de origen vegetal, aunque con dudas (Martínez Maganto et alii, 2001, 1224 y 1227); en cualquier caso, parece descartarse el empleo en la confección de dichas tintas negras de elementos metálicos -vitriolo-, no constituyendo por tanto tintes de naturaleza mineral. Da incluso la impresión de que la ausencia en el atramentum de partículas de hierro pueda ser la causa del mejor estado de conservación de estas inscripciones, ya que no se habrían producido fenómenos de oxidación, según algunos autores (Martínez Maganto et alii, 2001, 1228).

En el caso del rubrum, desconocemos analíticas similares que hayan podido caracterizar el tipo de pigmentos utilizados para la elaboración de estas tintas anaranjadas o rojas, más diluidas. A la espera de desarrollar estos trabajos en el futuro, parece evidente que posiblemente el empleo de minerales con componentes férricos sería una de las explicaciones, como quizás testimonie el depósito de almagra -óxidos de hierro- de la citada factoría murciana. Aunque tampoco debemos olvidar el potencial empleo de otras sustancias como quizás el propio cinabrio machacado, identificado en las cetariae de San Nicolás. El uso de cinabrio finamente pulverizado ha sido confirmado para la elaboración de pigmentos de color rojo bermellón -así como los hematites para el ocre rojo- en pintura parietal romana, como demostraron los análisis de unos cincuenta fragmentos de revestimientos altoimperiales de paredes procedentes de una excava- ción preventiva en el solar de la antigua Gades -c/ Santa María no 16- (Domínguez Bella, 2004, 201-203, figs. 3 D y 4 A). Su presencia en una fábrica salazonera quizás permita explicar otros usos alternativos, como el aquí planteado. En el futuro habrá que rastrear otras posibles sustancias vegetales -para el atramentum-.

\section{¿Veneno Para la PESCA?}

Por último, recordar el empleo entre las técnicas de pesca en la Antigüedad del veneno para la captura de peces. Así lo recuerda Opiano en su Halieutica cuando indica el empleo por parte de los pescadores de "un pernicioso veneno para los peces, y acarrean rápida muerte a las razas nadadoras"; una vez congregados los peces en una zona de escasa profundidad, "un hombre toma abundante arcilla blanca junto con la raíz que los médicos llaman ciclamen; mezclándolas con sus manos amasa dos tortas; y salta sobre las redes dentro del mar, y alrededor de las mismas cuevas y escondrijos de los peces unta el veneno de fétido olor del odioso ungüento, y contamina el mar...."; "y los peces acallan su murmullo y terrible tumulto, tras haber exhalado su quejumbroso aliento..."(IV, 647-683). Algo más tarde, ya en el s. III, Eliano, en su Historia de los Animales recuerda el empleo de plantas adormideras: "si un colmenero arroja a la laguna hojas de verbasco acigutre-, destruirá de la manera más sencilla a los renacuajos" $(\mathrm{I}, 58)$. Estas prácticas ancestrales han perdurado hasta bien entrado el s. XX, como encontramos reflejado en la obra de Rodríguez Santamaría, que nos recuerda el empleo de diversos productos como venenos, entre ellos el "cloruro de cal mezclado con aceite de vitriolo forman una pasta que mata a la pesca o, por lo menos, la ciega y la atonta; esto lo emplean en Levante..."; "En otros sitios usan la cocaína, llamada coca, en piedra o en polvo, mezclada con pan, y al comerla los peces, se atontan dando vueltas o se mueren..."; "también usan unas botellas de cloruro de cal; matapol, hierba venenosa que mata; el turbisco, planta que atonta, y el saquito o bolsa de cloruro de cal amarrado a una varita para coger los peces en donde están refugiados, y en Canarias una semilla de higuera de la Isla de Madera, que mezclada con peces y machacado todo hace una sustancia venenosa que mata los peces..." (Rodríguez Santamaría, 1923, 445-446). 
Es decir está claramente atestiguada la pesca en la Antigüedad recurriendo a venenos, por lo que parece usando hierbas o plantas de diversa naturaleza, aunque no se descarta el empleo de otras sustancias minerales (sulfatos, sales o ácidos). Quizás fuese ésta también una de las posibilidades para el empleo del polvo de cinabrio, aunque no debemos olvidar el carácter venenoso del mismo, dada su composición a base de mercurio y azufre, si bien nos es desconocida su posible acción inmediata sobre los peces, dada su poca solubilidad en el agua.

\section{NUEVAS LINEAS DE INVESTIGACIÓN: COLORANTES NO SOLO PARA LA PINTURA Y LOS TEXTILES}

La identificación arqueométrica en las fábricas de la c/ San Nicolás de Algeciras de micro-granos de cinabrio en dos molinos rotatorios manuales tardoantiguos ha permitido confirmar el empleo de este mineral en algunas actividades relacionadas con las actividades haliéuticas. No tiene mucho sentido el procesado de este mineral en las fábricas conserveras si el mismo no tuviese una relación directa con esta industria. El hallazgo de sustancias similares en otras fábricas conserveras hispanorromanas (Mazarrón) parece confirmar el habitual empleo de polvos colorantes en las industrias conserveras hispanorromanas.

Tratar de definir con claridad el uso que le fue dado al polvo de cinabrio obtenido por molturación en Traducta no es sencillo, ya que únicamente disponemos se su evidencia en los citados instrumentos de molienda. Una posibilidad es que fuese usado para la obtención de pigmentos para la pintura parietal, de la que no hay evidencias en las inmediaciones directas en los recintos fabriles pero que sabemos que debieron ornamentar las casas y otros edificios de la ciudad romana. El empleo de polvos de cinabrio, como ya hemos indicado, está confirmado en algunos ambientes domésticos del s. I d.C. en la ciudad de nueva planta erigida por Balbo el Menor en Gades (Domínguez Bella, 2004) o también en Asido (Domínguez-Bella y Morata, 2002). No obstante, consideramos que es más probable que tuviese alguna relación con las pesquerías y el ciclo de la industria conservera, de ahí que hayamos realizado una serie de reflexiones generales sobre el uso en general de las sustancias colorantes en este sector de la economía hispanorromana. Básicamente, como ilustramos en la figura 3, algunas de las posibilidades más sugerentes son: salsas piscícolas coloreadas, entintado de redes u otros aparejos, elaboración de pigmentos para tituli picti y quizás venenos para la pesca. No se excluyen, evidentemente, otros usos, habiéndonos limitado por cuestiones de espacio a desarrollar únicamente las citadas temáticas.

La elaboración de salsas piscícolas tintadas es una cuestión demostrada etnográficamente, como se ha detallado en algunos casos mediterráneos, como el de Volos en Grecia. Y para la Antigüedad también lo está, como verifican las inscripciones al laccatum tinctum recogidas en el CIL ya citadas. Independientemente de lo que realmente sea el Laccatum -posiblemente una salsa piscícola, pensamos- está claro que el producto fue coloreado, de ahí el adjetivo. También se ha discutido la posibilidad de que las inscripciones pintadas interpretadas hasta ahora como alusivas a los productos piscícolas de Tingis y Lixus sean en verdad "lejías" o productos lixiviados. Es decir obtenidos mediante el contacto de sólidos y líquidos -fracción líquida hipogástrica que provocaba la extracción vitamínica y de otras sustancias del preparado piscícola-, que en el primer caso serían "tintadas" y no "tingitanas". Es una posibilidad, propuesta por U. Ehmig (2007 a) muy sugerente y a valorar en el futuro, que permitiría explicar muchas cosas, como por ejemplo la anomalía de citar estas dos únicas ciudades, de manera mayoritaria y sin parangón, frente a otras más reputadas por sus salsas y totalmente ausentes o esporádicamente citadas en la literatura anforológica pintada. Estas nuevas interpretaciones harán retomar en el futuro algunas inscripciones consideradas hasta ahora como anómalas o de compleja lectura, como la de ¿Rub(rum) vetus? Annorum quattuor (CIL IV, 5597), que aunque relacionadas con la posible coloración de la salsa (Lagóstena, 2004, 214), habían pasado prácticamente desapercibidas a la investigación.

Proponemos que una de las razones de dichos tintados, además de atender los requerimientos organolépticos de los clientes - pensamos que sobre todo los visuales-, era emular el empleo de la sangre en las salsas. No olvidemos que el famoso garum de sangre o Haimation era, sin lugar a dudas, el más reputado, como destacan diversos 


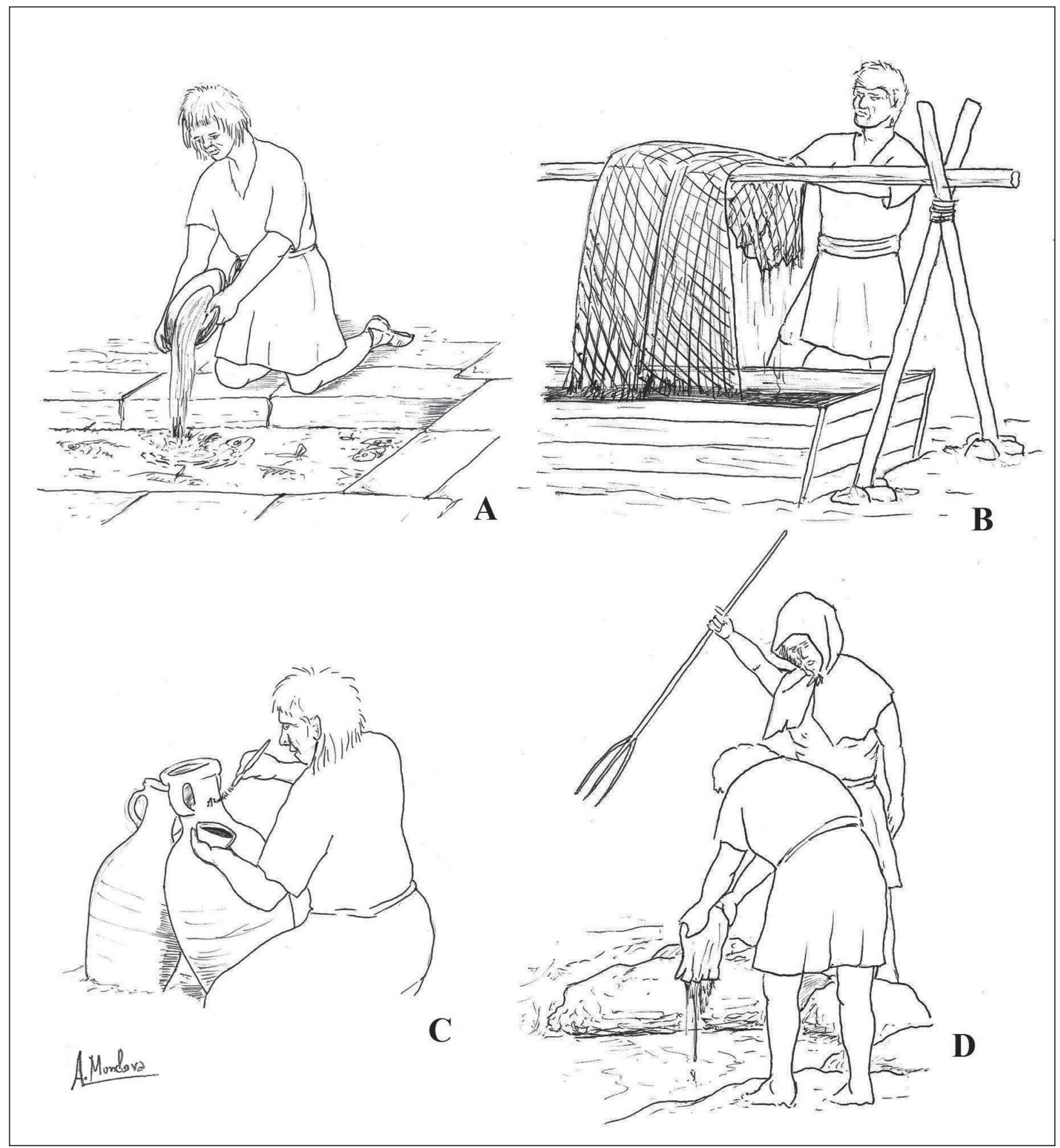

Figura 3.- Recreación del uso de colorantes en las fábricas conserveras hispanorromanas, tanto para tintar las salsas piscícolas (A), para el "entintado" de las redes y otros aparejos (B), para la elaboración de las inscripciones en las ánforas (C) y para la técnica de pesca con veneno (D). Dibujo de Antonio Monclova.

autores clásicos y la tradición literaria posterior (sintetizada en Curtis, 1991, 8, nota 11).

La segunda utilidad planteada es el empleo de colorantes para el tintado de las redes y aparejos, una práctica muy abundante en las pesquerías tradicionales ya que el tipo de fibras (lino, cáñamo o esparto) sufría mucho con las sales marinas y la grasa de los peces, y su impregnación con sustan- cias vegetales o minerales minimizaba su putrefacción y las convertía en más perdurables. Diversos productos eran habitualmente utilizados para ello como sabemos por cuestiones etnográficas, tanto la corteza de pino procesada como las almagras. Los diccionarios etnográficos sobre las artes de pesca permiten valorar retrospectivamente que esta práctica debió ser habitual en la Antigüedad, como confirman las referencias de 
Eliano en la Animalium Historia. Aunque subordinada y punto. De momento no está confirmada por falta de análisis químicos que hayan buscado estas sustancias en los escasos ejemplos de fibras conservadas ${ }^{2}$. Los únicos indicios arqueológicos disponibles son la citada bolsada de almagra de Mazarrón y estos restos de Traducta. Entre las propiedades del cinabrio figura su gran potencialidad fungicida y su milenario empleo para la conservación de restos óseos y de otra naturaleza (Martín-Gil et alii, 1995; Domínguez-Bella, 2010a y b), por lo que ésta es una de las posibilidades interpretativas más viables para el hallazgo de la c/ San Nicolás. No debemos olvidar que el trasiego de derivados de las coníferas era muy frecuente en las fábricas conserveras romanas, pues la pix -resina calentada- era habitualmente utilizada para impermeabilizar las ánforas (Bernal y Petit, 1991). Es decir, no sería raro por ello que además de la resina hubiesen utilizado la corteza machacada de los pinos u otros árboles para elaborar la mezcla bituminosa ya citada que conocemos en época moderna y contemporánea para el entintado de las redes. En cualquier caso este es un campo totalmente novedoso de la investigación, en el cual futuras analíticas de residuos sin duda aclararán el panorama. Baste citar, por ejemplo, la reciente constatación de azufre, a través de análisis molecular, en algunos revestimientos internos de ánforas olearias del tipo Africana I procedentes del Ródano, un producto también fungicida y característico por constituir un buen agente de impermeabilización (Garnier, Silvino y Bernal, 2011). Es decir, tampoco debemos descartar el empleo de otros productos para mejorar la conservación del instrumental de pesca realizado en fibras vegetales.

En tercer lugar traemos a colación la importancia de pigmentos para la elaboración de pintura para elaborar los tituli picti en las ánforas y otros envases de conservas piscícolas que debieron ser convenientemente etiquetados, siguiendo el mos italicus. Tinta negra -atramentum- posiblemente con carbones vegetales, y colorante rojo -rubrum- con sustancias no bien conocidas por el momento. Esta es la segunda posibilidad interpretativa para los granos de cinabrio identificados en
Algeciras. Una dinámica que es la habitual de este producto machacado, su empleo en pintura, de ahí que también sería lógico que se hubiese recurrido a él para hacer las inscripciones en rojo presentes en muchas de nuestras ánforas béticas, aunque porcentualmente sean inferiores a las escritas con cálamos en negro. Hasta la fecha no ha sido localizada ninguna en el yacimiento con un titulus pictus en rojo, para poder ser analizada arqueométricamente.

Por último, se llama la atención sobre el uso de venenos para la pesca, citados por diversos autores clásicos, de manera que es probable el empleo de plantas u otras sustancias en nuestras fábricas conserveras, las cuales han dejado escasas huellas. Al constituir el cinabrio un producto nocivo para la salud, por su elevado porcentaje de mercurio, parece lógico descartar su empleo para la ejecución de venenos píscicolas.

Por el momento las evidencias disponibles son mínimas, limitadas a los datos procedentes de c/ San Nicolás de Algeciras y de la c/ Nueva Callejón de la Sal de Mazarrón, ambas de época tardorromana. Estamos seguros que una mirada atenta al registro de muchas de nuestras fábricas pesqueras deparará suculentas novedades en los próximos años. Además del colorante púrpura, tan importante en las fábricas conserveras romanas y cuya presencia es cada vez más abundante en las cetariae hispanas (García Vargas, 2004; Bernal, Sáez y Bustamante, 2011), debemos tener presente la existencia de otras materiales colorantes, rojas, negras y de diversa colorimetría, que debieron ser usadas por los piscatores romanos en los complejos procedimientos fabriles cargados de la sabiduría que decenas de generaciones aportaron a lo largo de la Historia preislámica.

\section{Agradecimientos}

Queremos dejar constancia de la amabilidad de Dimitra Mylona al proporcionarnos las referencias etnográficas sobre las salsas piscícolas tintadas de Volos. Y a Enrique García Vargas por sus siempre útiles y doctos comentarios durante la lectura del manuscrito original.

2 Esta es una de las líneas de investigación en desarrollo actualmente a través del proyecto SAGENA (www.sagena.es) 


\section{Bibliografía}

AGÜERA MARTÍNEZ, S. e INIESTA SANMARTÍN, A. (1998): "Actuación arqueológica en el casco urbano del Puerto de Mazarrón: el solar de c/ Nueva $\mathrm{n}^{\circ} 11$ - callejón de la Sal", Libro de Resúmenes de las IX Jornadas de Arqueología Regional (Murcia, mayo 1998), Murcia, p. 46.

AGUILERA MARTÍN, A. (2004): "Sistematización de los tituli picti anfóricos para la Base de Datos CEIPAC", en J. Remesal ed., Epigrafia anfórica, Col.lecció Instrumenta17, Barcelona, pp. 105126.

ALFARO GINER, C. (2010): "Fishing Nets in the Ancient World: the historical and archaeological evidence", en Bekker-Nielsen, T. y Bernal, D., eds., Ancient Nets and Fishing Gear, Monographs of the Sagena Project 2, Aarhus y Cádiz, pp. 5581.

ARÉVALO, A. y BERNAL, D. (2007, eds.): Las cetariae de Baelo Claudia. Avance de las investigaciones arqueológicas en el barrio meridional (2000-2004), Sevilla.

ATHANASSOPOULOS, M. (1926): "Notes sur la faune en Grèce", Bulletin de l'Institut Océanographique 480, Mónaco, pp. 1-7.

BEKKER-NIELSEN, T. (2003): Ancient fishing and fish processing in the Black Sea region, Black Sea Studies 2, Aarhus.

BEKKER-NIELSEN, T. y BERNAL, D. (2010): Ancient Nets and Fishing Gear, Monographs of the Sagena Project 2, Aarhus y Cádiz.

BERNAL, D. (2007): “Algo más que garum. Nuevas perspectivas sobre la producción de las cetariae hispanas al hilo de las excavaciones en la c/ San Nicolás (Algeciras, Cádiz)", en Lagóstena, L., Bernal, D. y Arévalo, A. eds., Cetariae. Salsas y salazones de pescado en Occidente en la Antigüedad, BAR 1686, Oxford, pp. 93-107.

BERNAL, D. (2009, ed.): Arqueología de la Pesca en el Estrecho de Gibraltar. De la Prehistoria al fin del Mundo Antiguo, Monografías del Proyecto Sagena 1, Madrid.

BERNAL, D. (2012, ed.): Las factorías de salazones de Traducta. Primeros resultados de las excavaciones arqueológicas en la c/ San Nicolás de Algeciras (2001-2006), Cádiz, en prensa.

BERNAL, D., ALONSO, C. y GRACIA, J. (2011): "De la acuicultura en Baetica. A propósito de la posible piscina-vivero del yacimiento haliéutico del Cabo Trafalgar (Cádiz)", Zephyrus LXVII, pp. $145-160$.
BERNAL, D., JIMÉNEZ-CAMINO, R., LORENZO, L., TORREMOCHA, A. y EXPÓSITO, J.A. (2003): "Las factorías de salazones de Iulia Traducta. Espectaculares hallazgos arqueológicos en la calle San Nicolás 3-5 de Algeciras", Actas de las VII Jornadas de Historia del Campo de Gibraltar, Almoraima 29, Algeciras, pp. 163-183.

BERNAL, D. y PETIT, M.A. (1991): “Caracterización de resinas en ánforas romanas por Cromatografía de Gases. Resultados y aplicaciones en España”, Alebus 4-5, Elda, pp. 83-98.

BERNAL, D., SÁEZ, A.M. y BUSTAMANTE, M. (2011): "Púrpura y pesca en el Gadir tardopúnico. La fosa-conchero de desechos haliéuticos de la c/ Luis Milena (San Fernando, Cádiz)", Purpureae Vestes III, pp. 157-180.

BOTTE, E. (2009): Salaisons et sauces de poisons en Italie du Sud et en Sicile durant l'Antiquité, Collection du Centre Jean Bérard, 31, Nápoles.

CERRI, L. (2006): "Tituli picti di Lixus e Tingis", L'Africa Romana XVI (Rabat, 2004), pp. 21752182.

CHIC GARCÍA, G. (2002): "Degustatio o Recognitio", en L. Rivet y M. Sciallano eds., Vivre, produire et échanger: reflets méditerranéens. Mélanges offerts à Bernard Liou, Archéologie et Histoire Romaine, 8, Éditions Monique Mergoil, Montagnac, pp. 335-342.

CURTIS, R.I. (1991): Garum and Salsamenta. Production and commerce in material medica, Studies in ancient Medicine, 3, Brill.

CURTIS, R.I. (2001): Ancient Food Technology, Technology and Change in History 5, Brill.

DOMÍNGUEZ BELLA, S. (2004): “Pinturas murales romanas en la Neapolis gaditana (Cádiz). Análisis de pigmentos minerales y caracterización de estucos”, en Feliù, M.J., Martín, J., Edreira, M.C., Fernández Lorenzo, M. C., Martínez Brell, M.P., Gil, A. y Alcántara, R. eds., Avances en Arqueometría, Universidad de Cádiz, pp. 201207.

DOMÍNGUEZ-BELLA, S. (2010a): “Aplicaciones de las técnicas experimentales y la mineralogía a la arqueometría. Los pigmentos de cinabrio del dolmen de Alberite I, Villamartín (Cádiz)", en Domínguez-Bella, S., Ramos Muñoz, J., Gutiérrez López, J.M. y Pérez Rodríguez, M., eds., Minerales y Rocas en las Sociedades de la Prehistoria. Grupo HUM-440, Universidad de Cádiz, pp. 235-244. 
DOMÍNGUEZ-BELLA, S. (2010b): "Rojo y verde: los minerales en la vida y la muerte del megalitismo neolítico", IV Encontro Arqueolóxico do Barbanza. Boiro, Libro de Conferencias. DVD $\mathrm{n}^{\circ}$ 5, Ed.: Centro Arqueolóxico do Barbanza. A Coruña.

DOMÍNGUEZ BELLA, S. (2012): "Estudio arqueométrico e implicaciones sobre el origen y utilización de los molinos de mano de la factoría de c/ San Nicolás. Primeros resultados", en Bernal, D. (ed.): Las factorías de salazones de Traducta. Primeros resultados de las excavaciones arqueológicas en la cl San Nicolás de Algeciras (20012006), Cádiz, en prensa.

DOMÍNGUEZ-BELLA, S. y BERNAL-CASASOLA, D. (2011): "Fish-Based subproducts in Late Antiquity. Archaeometric and archaeological evidence from the Fish Factories at Traducta (Algeciras, Cádiz, Spain)", en Turbanti-Memmi, I., Proceedings of the $37^{\text {th }}$ International Symposium on Archaeometry, pp. 453-458. Londres.

DOMÍNGUEZ-BELLA, S. y MORATA CÉSPEDES, D. (1996): "Caracterización mineralógica y petrológica de algunos objetos del ajuar y de los recubrimientos de las paredes y suelos de la cámara (materiales líticos y ocres)", en Ramos Muñoz, J. y Giles Pacheco, F. (Eds. y coord.), 1996, El dolmen de Alberite (Villamartín). Aportaciones a las formas económicas y sociales de las comunidades neolíticas en el noreste de Cádiz, Cádiz, pp. 187206.

DOMÍNGUEZ-BELLA, S. y MORATA CÉSPEDES, D. (2002): "Mineralogical and chemical characterization of roman wall paintings from MedinaSidonia, Cádiz, Spain", en Archaeometry 98. Proceedings of the 31st International Symposium on Archaeometry (1998, Budapest), ed. E. Jerem y K.T. Biró, BAR International Series 1043 (II), Oxford, pp. 715-722.

EHMIG, U. (2003): Die Römischen Amphoren aus Mainz, Frankfurter Archäologische Schriften 4, Möhnesee.

EHMIG, U. (2007 a): "Tituli picti auf Amphoren in Köln”, Kölner Jahrbuch 40, pp. 215-322.

EHMIG, U. (2007 b): Die Römischen Amphoren im Umland von Mainz, Frankfurter Archäologische Schriften 5, Wiesbaden.

ÉTIENNE, R. y MAYET, F. (2002): Salaisons et sauces de poisson hispaniques, París.

FERNÁNDEZ IZQUIERDO, A. (1984): Las ánforas romanas de Valentia y de su entorno marítimo, Valencia.
GALLART, L., ESCRICHE, I. y FITO, P. (2004): La salazón de pescado, una tradición en la dieta mediterránea, Universidad Politécnica de Valencia, Valencia.

GARCÍA VARGAS, E. (2004): "Las pesquerías de la Bética durante el Imperio romano y la producción de púrpura", Purpureae Vestes I, pp. 219-235.

GARCÍA VARGAS, E. (2006): "Garum sociorum: pesca, salazones y comercio en los litorales gaditano y malacitano (época altoimperial romana)", Setúbal Arqueológica 13, pp. 39-56.

GARNIER, N., SILVINO, T. y BERNAL-CASASOLA, D. (2011): "L'identification du contenu des amphores: huile, conserves de poissons et poissage”, SFECAG. Actes du Congrès d'Arles, pp. 120, pp. 397-416.

GUTIÉRREZ MAS, J.M., MARTÍN ALGARRA, A., DOMÍNGUEZ-BELLA, S. y MORAL CARDONA, J.P. (1991): Introducción a la geología de la provincia de Cádiz. Servicio de Publicaciones de la Universidad de Cádiz. Cádiz.

JIMÉNEZ-CAMINO, R. y BERNAL, D. (2007): "Redescubriendo a Traducta. Reflexiones sobre su topografía urbana y su secuencia ocupacional (ss. I - VII d.C.)", Anuarios de Arqueología Cordobesa 18, Córdoba, pp. 157-200.

LAGÓSTENA BARRIOS, L. (2004): "Las ánforas salsarias de Baetica. Consideraciones sobre sus elementos epigráficos”, en J. Remesal ed., Epigrafia anfórica, Col.lecció Instrumenta 17, Barcelona, pp. 197-217.

MAHDIHASSAN, S. (1984): “Tan, cinnabar, as drug of longevity prior to alchemy", American Journal of Chinese Medicine, 12, pp. 50-54.

MARTIN GIL, J, MARTIN GIL, F. J, DELIBES DE CASTRO, G, ZAPATERO, P. y SARABIA, F.J. (1995): "The first known use of vermillion". Experimentia 51 (8): 759-761.

MARTÍN KILCHER, S. (2002): "Lucius Urittius Verecundus, négociant à la fin du Ier siècle, et sa marchandise découverte à Mayence", en L. Rivet y M. Sciallano eds., Vivre, produire et échanger: reflets méditerranéens. Mélanges offerts à Bernard Liou, Archéologie et Histoire Romaine, 8, Éditions Monique Mergoil, Montagnac, pp. 343-353.

MARTÍNEZ ALCALDE, M. y INIESTA SANMARTÍN, A. (2007): Factoría romana de salazones. Guía del Museo Municipal de Mazarrón, Mazarrón. 
MARTÍNEZ MAGANTO, J., FERRERO CALABUIG, J.L., ROLDÁN GARCÍA, C. y ÁLAMO SERRANO, A. (2001): "Los tituli picti, estudio sobre el atramentum y su composición", Ex Baetica Amphorae, pp. 1221-1229.

MAHDIHASSAN, S. (1984): “Tan, cinnabar, as drug of longevity prior to alchemy", American Journal of Chinese Medicine, 12, pp. 50-54.

MYLONA, D. (2008): Fish-eating in Greece from the fifth century B.C. to the seventh century A.D., B.A.R. 1754, Oxford.

PLINIO EL VIEJO. Historia Natural, Lapidario. Libros XXXVI y XXXVII. Traducción de Avelino Domínguez e Hipólito B. Riesco. 1993. Alianza Editorial. Madrid.

PONSICH, M. (1988): Aceite de oliva y salazones de pescado. Factores geo-económicos de Bética y Tingitania, Madrid.

QUILLON, K. (2011): “Les inscriptions peintes sur amphores à sauce et salaison de poisson hispaniques", en Djaoui, D., Greck, S. y Marlier, S., Arles-Rhône 3. Le naufrage d'un chaland antique dans Le Rhône, enquete pluridisciplinaire, Actes Sud, pp. 107-109.
RODRÍGUEZ SANTAMARÍA, B. (1923): Diccionario de artes de pesca de España y sus posesiones, Madrid.

SÁÑEZ REGUART, A. (1793): Diccionario histórico de los artes de la pesca nacional, Madrid. Edición fac-simil, 5 tomos, Editorial Maxtor, Valladolid.

SLIM, L., BONIFAY, M., PITON, J. y STERNBERG, M. (2007): "An example of fish salteries in Africa Proconsularis: the officinae of Neapolis (Nabeul, Tunisia)", en Lagóstena, L., Bernal, D. y Arévalo, A. eds., Cetariae. Salsas y salazones de pescado en Occidente en la Antigüedad, BAR 1686, Oxford pp. 21-44.

VAN NEER, W. y PARKER, T. (2008): "First archaeozoological evidence for haimation, the invisible garum", Journal of Archaeological Science 35, 7, pp. 1821-1827. 
